\title{
Study on the Weak Parametrically Excited Mathieu Stability of Submerged Monitoring Platform
}

\author{
Kong-De HE ${ }^{1}$, Xu-Guang XIE ${ }^{1, a^{*}}$, Zi-Fan FANG ${ }^{1}$, Wei-Hua YANG ${ }^{1}$, \\ Xue-Hui HE${ }^{1}$, Kong-De $\mathrm{HE}^{2, \mathrm{~b}}$ \\ ${ }^{1}$ Mechanic and Material College, China Three Gorges University, Yichang, China \\ ${ }^{2}$ Hubei Key Laboratory of Hydroelectric Machinery Design \& Maintenance, China Three Gorges \\ University, Yichang, China \\ a956039550@qq.com, bhekongde@ctgu.edu.cn \\ ${ }^{*}$ Corresponding author
}

\begin{abstract}
Keywords: Submerged monitoring platform; Stability; Weak parametrically excited; Mathieu equation
\end{abstract}

\begin{abstract}
Aimed at the heaving pitch coupling stability problem for underwater mooring monitoring platform under the action of a nonlinear flow field, the heaving pitch coupled equations of motion were established in consideration of initial stability height and drainage volume change; The stability transition curve of underwater mooring monitoring platform when the frequency ratio is different were obtained by application of Floquet theory, virtual quality standard method and deformation parameters method, studied the stability of weak parametric excitation characteristics, determine the stability and instability regions, and validate the characteristics by the time history response.
\end{abstract}

\section{Introductions}

Submerged monitoring platform is mainly used for supporting underwater monitoring, detection equipment and installation cross section parallel to the river, to the implementation of the regional security detection and scanning sonar installed in the above and other detection devices. It requires a certain depth long cloth on the underwater environment, continuous measurement of laying areas, in order to obtain a stable detection signal and need this type of device itself has stable dynamic characteristics in the external flow field, and therefore, submerged monitoring platform of stability has important significance. The working environment of the submerge monitoring platform is a new type of device, which is a new type of device, which can be subjected to the uncertain fluid forces from the outside flow field in the working process, and the working environment is complex. In the complex external environment load will be substantial under the action of coupled motion of heave and pitch, and coupling motion makes it hard to obtain accurate monitoring results, and even lead to the platform body overturning instability, so the study of the submerge monitoring platform in the external environment of stability, the complex role of the safety assessment body platform, with engineering and theoretical significance is very important. [1]

\section{The Coupled Motion Equations of Heave and Pitch}

Submerged monitoring platform by the mooring, float, and other accessories installed in the above composition, as shown in Fig1.Not only the work of its waters affected by environmental dynamics in addition to the load, but also affected the shape of the structure itself, the impact of environmental loads the largest is the flow field velocity, direction, and float deployment depths 2. The main force of the flow field environment float suffered at a certain depth of water is fluid force and circle resistance, flow perpendicular to the axial direction of the floating body fluid force and circle resistance are the biggest, velocity parallel to on the floating body in the axial direction of the flow around a minimum resistance, simplified mechanical model and principal body axes after being subjected to load flow field, as shown in Fig2 3. In the mid-point floating body axis of the 
coordinate origin, the floating body axis of the $\mathrm{X}$-axis, Y-axis is perpendicular to the floating body axis pointing to the water and flow field velocity forward Z-axis direction, the platform around the $\mathrm{X}$ axis of rotation for pitching motion, $\mathrm{Y}$ motion to heave motions, when the platform under the action of flow field occurrence of heave and pitch motions, its position will change, with the change from co-rotational coordinate system $\mathrm{O}-\mathrm{XYZ}$ to $\mathrm{O}^{\prime}-\mathrm{X}^{\prime} \mathrm{Y}^{\prime} \mathrm{Z}$ '.

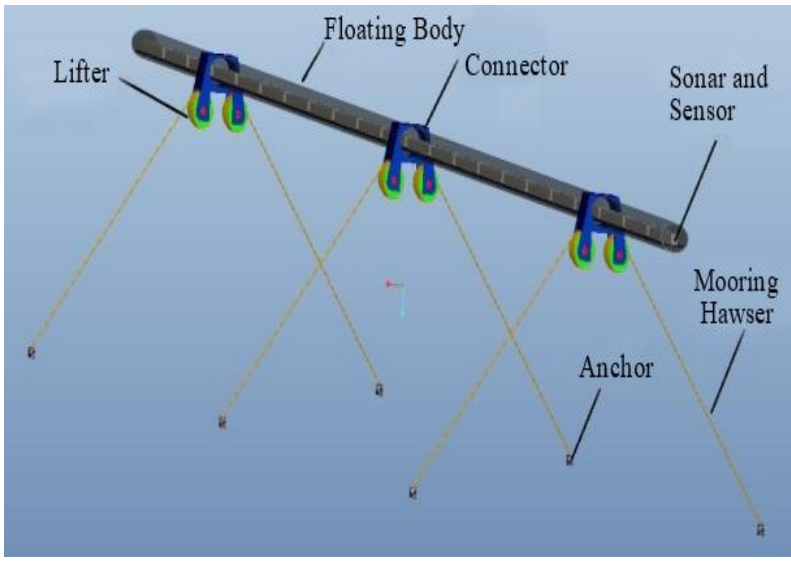

Fig. 1. Schematic diagram

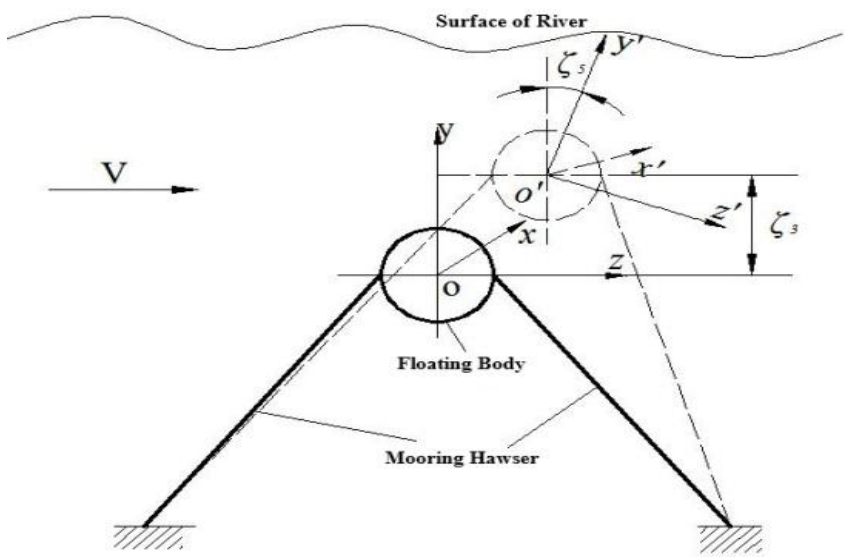

Fig. 2. Schematic diagram

The heave motions as amplitude periodic motion $\xi_{a}$, the introduction of variable substitution $\tau=\omega_{\mathrm{a}} \mathrm{t}$, to:

$$
\xi_{5}+\delta \xi_{5}+(\gamma-\beta \cos t) \xi_{5}=0
$$

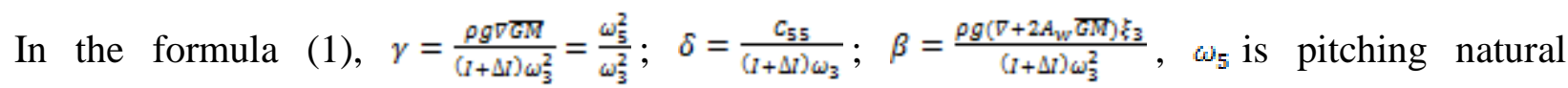
frequency, $\omega_{\mathrm{a}}$ is heave natural frequency.

\section{Weak Effect Parameters Mathieu Equation}

Since the underwater monitoring platform mooring pontoon located a certain depth under water, at a flow rate of the flow field little change in the situation to ensure a certain degree of stability, when the heave amplitude changes. $\zeta_{a}$ is small, equation (1) can approximate solution application of the deformation parameter method to solve 4 , it is assumed to approximate the small parameter $\beta$, provided:

$$
\gamma=m^{2}+\beta \delta_{1}+\beta^{2} \delta_{2}+\cdots
$$

Assuming that ${ }^{\delta}$ can be expanded into the following algebraic form:

$$
\delta=\beta \delta_{1}+\beta^{2} \delta_{2}+\cdots
$$

The solution of the equation (4) can be expressed as the following form:

$$
\xi_{5}=\xi_{50}+\beta \xi_{51}+\beta^{2} \xi_{52}+\cdots
$$

Equation (2), (3), (4) into equation (1), the power of comparison performed $\beta$ can be obtained:

$$
\begin{aligned}
& \xi_{50}+m^{2} \xi_{50}=0 \\
& \xi_{51}+m^{2} \xi_{51}=-\delta_{1} \xi_{50}^{*}-\delta_{1} \xi_{50}+\xi_{50} \cos \tau \\
& \xi_{52}+m^{2} \xi_{52}=-\delta_{2} \xi_{50}^{*}-\delta_{1} \xi_{51}^{x}-\delta_{1} \xi_{51}-\delta_{2} \xi_{50}+\xi_{51} \cos \tau
\end{aligned}
$$


Through the calculation of $\beta, \gamma$ value, and using them as transverse and longitudinal coordinates into plane curve, the standard Mathieu equation has periodic solutions in the $\gamma-\beta$ curve, $\gamma-\beta$ plane by Mathieu equation curve is divided into two parts, respectively, for the stable region and unstable region, based on the $m=0,1,1 / 2$,analysis of three cases.

(1) $m=0$, Solving equation (3) to (5) can be obtained:

$$
\xi_{50}=\mathrm{a},(\mathrm{a} \text { are constants) }
$$

$$
\xi_{51}=-a \cos \tau
$$

$$
\xi_{52}^{*}=-\delta_{1} a \sin \tau-\sigma_{2} a-\frac{a}{2}(1+\cos 2 \tau)
$$

Of the formula (13), so $\sigma_{2}=-1 / 2$, can eliminate the long-term period to solve the formula (13) in the solution for:

$$
\xi_{52}=a\left(\delta_{1} \sin \tau+\frac{1}{g} \cos 2 \tau\right)
$$

Pitch angle can be solved Periodic Solutions $\xi_{5}$ as follows:

$$
\xi_{5}=a-a \varepsilon \cos \tau+a\left(\delta_{1} \sin \pi+\frac{1}{8} \cos 2 \pi\right) \gamma^{2}
$$

Excessive curve equation:

$$
\beta=-\frac{1}{2} \gamma^{2}
$$

(2) $m=1$, Solving equation (5):

$\xi_{50}=a \cos \tau+b \sin t \quad(a, b$ are constants $)$

Of formula (14) into the formula (6), to:

$\xi_{51}+m^{2} \xi_{51}=\left(\delta_{1} a-\sigma_{1} b\right) \sin \tau-\left(\sigma_{1} a-\delta_{1} b\right) \cos \tau+\frac{a}{2}+\frac{1}{2}(a \cos 2 \tau+$

$b \sin 2 \tau)$

Elimination of secular terms from the formula (15), to: $\delta_{1}^{2}+\sigma_{1}^{2}=0$

Available $\delta_{1}=\sigma_{1}=0$, solve the formula (18) can be obtained:

$\xi_{51}=\frac{a}{2}-\frac{1}{6}(a \cos 2 \tau+b \sin 2 \tau)$

Of formula (15), (16) into (7) can be obtained:

$\xi_{5}=$

$a \cos \tau+b \sin \tau+\gamma\left[\frac{\alpha}{2}-\frac{1}{6}(\cos 2 \tau+b \sin 2 \tau)\right]-\gamma^{2}\left\{\frac{1}{18}\left[\left(\sigma_{1} a+2 \delta_{1} b\right) \cos 2 \tau+\right.\right.$

$\left.\left.\left(2 \delta_{1} a-\sigma_{1} b\right) \sin 2 \tau\right]+\frac{\sigma_{1}}{2} a-\frac{1}{96}(a \cos 3 \tau+b \sin 3 \tau)\right\}$

Excessive curve: 


$$
\begin{aligned}
& \frac{5}{12} \gamma^{2}=-2(\beta-1) \pm \sqrt{9(\beta-1)^{2}+5 \delta^{2}} \\
& \text { (3) } m=1 / 2 \text {, Solving equation }(8): \\
& \xi_{50}=a \cos \frac{\tau}{2}+b \sin \frac{\tau}{2}(a, b \text { are constants })
\end{aligned}
$$

Solving equation (6):

$$
\xi_{51}=-\frac{1}{4}\left(a \cos \frac{a}{2} \tau+b \sin \frac{a}{2} \tau\right)
$$

Solving equation (7), elimination of secular terms and to achieve the accuracy of the gamma ${ }^{\wedge} 2$ order, the boundary equation can be obtained:

$$
\begin{aligned}
& \gamma^{2}=(10-8 \beta) \pm 8 \sqrt{\frac{a}{2}-\frac{1}{4} \delta^{2}-2 \beta} \\
& \xi_{5}= \\
& a \cos \tau+b \sin \tau-\frac{1}{4} \gamma\left(a \cos \frac{a}{2} \tau+b \sin \frac{a}{2} \tau\right)-\frac{1}{16} \gamma^{2}\left[\left(2 \sigma_{1} a+3 \delta_{1} b\right) \cos \frac{a}{2} \tau-\right. \\
& \left.\frac{1}{16}\left(3 \delta_{1} a+2 \sigma_{1} b\right) \sin \frac{a}{2} \tau+\frac{1}{48}\left(a \cos \frac{5}{2} \tau+b \sin \frac{5}{2} \tau\right)\right]
\end{aligned}
$$

\section{Submerged design parameters}

According to submerged monitoring platform design requirements, determine the relevant design parameters are shown in Tab1.

Tab. 1 Design Parameters

\begin{tabular}{|l|c|}
\hline \multicolumn{1}{|c|}{ Parameter name } & Value \\
\hline Floating body length/m & 12.5 \\
\hline Floating body diameter/m & 0.2 \\
\hline Mooring diameter/m & 0.006 \\
\hline Inertia force coefficient & 1 \\
\hline Water depth/m & 63.2 \\
\hline From the river to the floating body/m & 10 \\
\hline Viscous drag coefficient & 0.4 \\
\hline River density/kg/m3 & 1000 \\
\hline The average flow rate of the river at the floating body/m / s & 2.52 \\
\hline Lift coefficient & 0.07 \\
\hline Floating body weight/ kg & 365.75 \\
\hline Floating bulk modulus of elasticity/Mpa & 17500 \\
\hline The dimensionless damping ratio of the structure & 0.01 \\
\hline
\end{tabular}




\section{Solution of Natural Frequency of Heave and Pitch}

Modeling. For submerged monitoring platform parameters stability analysis and time history poise of weak parameters under the Excitation research is needed to solve it heave, pitch natural frequency to determine the value of the parameter, the use of fluid-solid coupling analysis of flow the virtual mass force field method principles loaded onto the model, considering the difficulty of solving, finite element surface model mooring line monitoring platform, according to the design parameters and requirements, mooring the floating body adopts shell elements, using mooring lines cable element simulation, consider the tension, twisted by the mechanical properties of finite element coupling between flow line and plane model shown in Fig 3, Fig 4 is an enlarged view.5

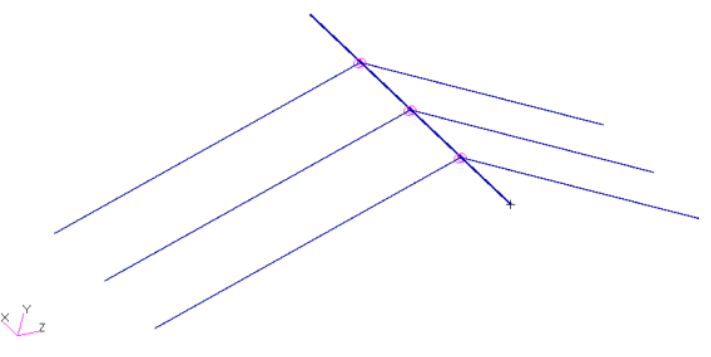

Fig.3. The finite element model

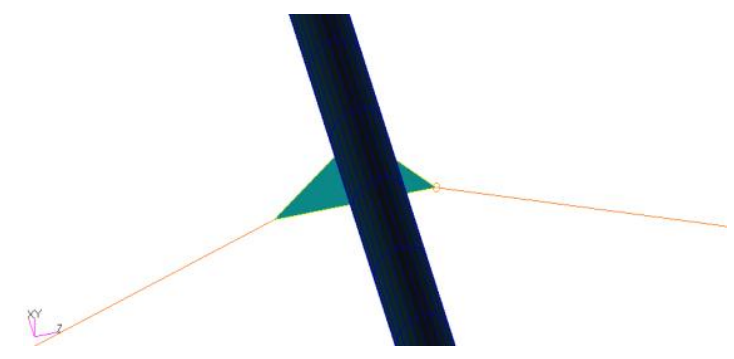

Fig. 4. Local Magnification

Definition of Coupling Surface. Solving fluid-structure coupling finite element model, we need to define the coupling between the platform and the flow field only defines the coupling flow field and platform, the platform movement and interaction of the flow field can be coupled to motion along the surface of the platform to take the coupling face to face interaction coupling force transmission medium between the flow field and platform, coupled defined surface shown in Fig 5, the finite element software solution process, the need to ensure that the closed nature of the coupling surfaces, the coupling surface normal direction point outside flow field, and the platform surface to be located within the coupling flow field grid, coupling relationship will work, specifically define the relationship shown in Fig 6.

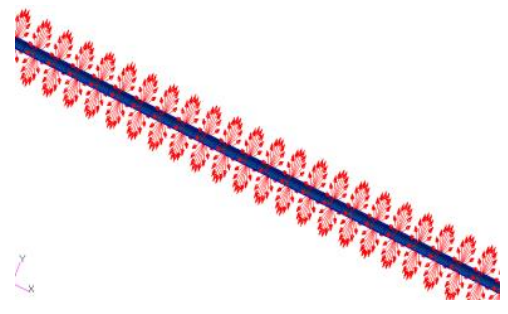

Fig. 5 Definition of coupling surface

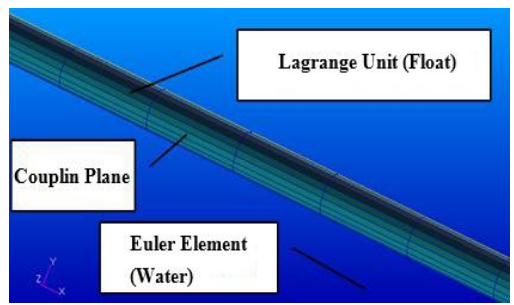

Fig. 6 Normal direction of coupling surface

Flow Calculation Coupled Modes. Solid coupling analysis of the above definition flow must be defined in order to achieve in the software, the calculation of mooring monitoring platform feature in a certain depth when the value of fluid solid coupling analysis can be achieved by modifying the model data card MFLUID and ELIST calculated using virtual quality of Msc Nastran software method. MFLUID cards are used to define the nature of the water, as shown in Tab 2; the ELIST card is used to define the fluid solid coupling surface, as shown in Tab 3: 
Table 2. MFLUID Card

\begin{tabular}{|c|c|c|c|c|c|c|c|c|c|}
\hline Number & 1 & 2 & 3 & 4 & 5 & 6 & 7 & 8 & 9 \\
\hline $\begin{array}{c}\text { Card } \\
\text { Name }\end{array}$ & MFLUID & SID & CID & ZFS & RHO & ELIST1 & ELIST2 & PLANE1 & PLANE2 \\
\hline Parameter & $\begin{array}{c}\text { Default } \\
\text { Value }\end{array}$ & 1 & $\begin{array}{c}\text { Default } \\
\text { Value }\end{array}$ & 10 & 1000 & 11 & $\begin{array}{c}\text { Default } \\
\text { Value }\end{array}$ & $\mathrm{n}$ & $\mathrm{n}$ \\
\hline
\end{tabular}

ZFS which is used to define the depth, RHO is used to define the density of water.

Table 3. ELIST Card

\begin{tabular}{|c|c|c|c|c|c|c|c|}
\hline Number & 1 & 2 & 3 & 4 & 5 & 6 & 7 \\
\hline $\begin{array}{c}\text { Card } \\
\text { Name }\end{array}$ & ELIST & LID & E1 & E2 & E3 & E4 & etc \\
\hline Parameter & $\begin{array}{c}\text { Default } \\
\text { Value }\end{array}$ & 11 & 1 & Thru & 1620 & $\begin{array}{c}\text { Default } \\
\text { Value }\end{array}$ & $\begin{array}{c}\text { Default } \\
\text { Value }\end{array}$ \\
\hline
\end{tabular}

On tact with the flow field is 1-1620, since the platform completely in the water that is all the elements are in contact with water, consistent with the coupling surface shown in Fig5.

Calculation Results. Based on the above mentioned above, the analysis is presented to obtain the results of the vertical swing, the shaking mode and the mode shape of the platform under the $10 \mathrm{~m}$ deep water.

As can be seen from the results, submerged monitoring platform heave natural frequency is $0.0235 \mathrm{HZ}$; pitching natural frequency is $0.0079 \mathrm{HZ}$.

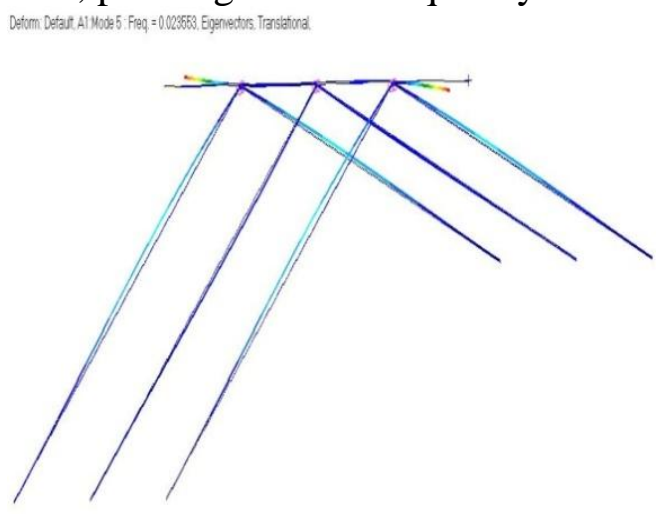

Fig. 7. Water drop swing type

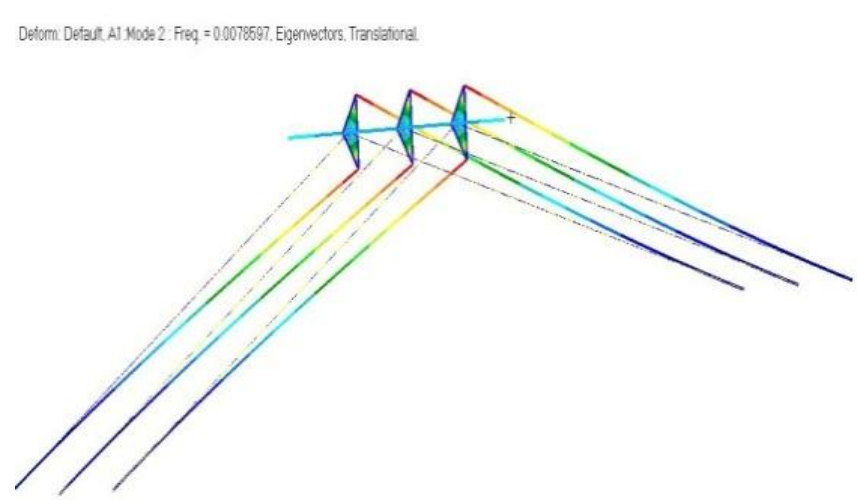

Fig. 8. Underwater longitudinal vibration model

\section{Numerical Verification}

Stability Criteria. Equation (4) is a linear differential equation containing periodic coefficients, according to the Floquet theory cycle coefficient linear differential equations, boundary equation function curve function of the plane into two regions, namely stable region and unstable region 6 .

According to the transition curve equation, formula (4) is a standard damped Mathieu equation, the stability characteristics of Mathieu equation parameters determined by $\gamma, \beta 7$, when $\delta=0$, is undammed Mathieu equation, were drawn undammed and over curve of different damping, undammed stability region and unstable region map, Fig 9 is no excessive damping curve, Fig 10 for damping for different values of transition curve. 
As can be seen from Fig 9, submerged monitoring platform undammed Mathieu equation stability map by the two curves enclosed, the inside curve is the unstable region, the external curve is stability in the region; damping ratio when changing the system from Fig. 10 can be seen as the damping increases, excessive curve equation instability range into the area around gradually reduced, gradually increasing the range of stability, it can be controlled by changing submerged monitoring platform heave displacement, vertical roll angle range, such as external monitoring platform mooring increase helical damping side, cross-sectional shape changing platform floating body, increasing the cross-sectional diameter and the like.

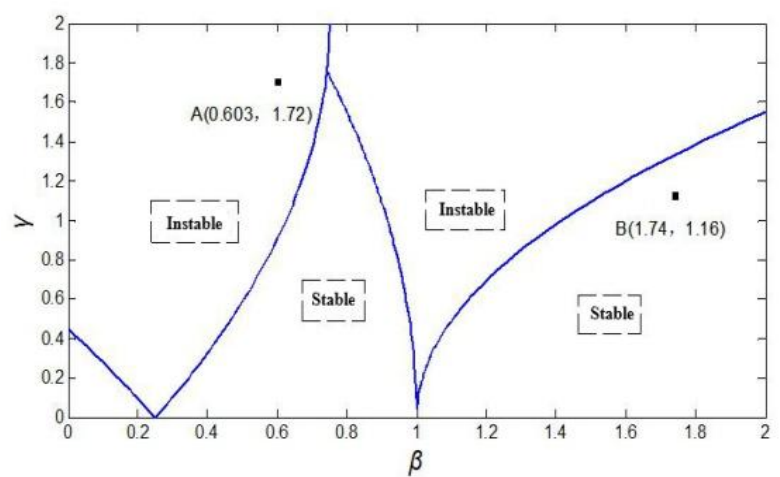

Fig. 9. Undamping stability map

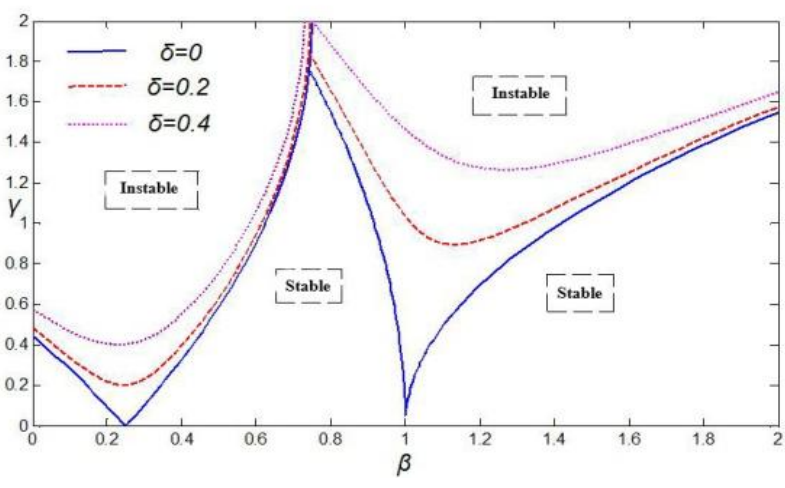

Fig. 10. Different damping stability maps

Time History Analysis. In order to verify the stability analysis, the stability of the region and instability in the region were taken points $\mathrm{A}$ and $\mathrm{B}$ point mooring monitoring platform time history analysis.

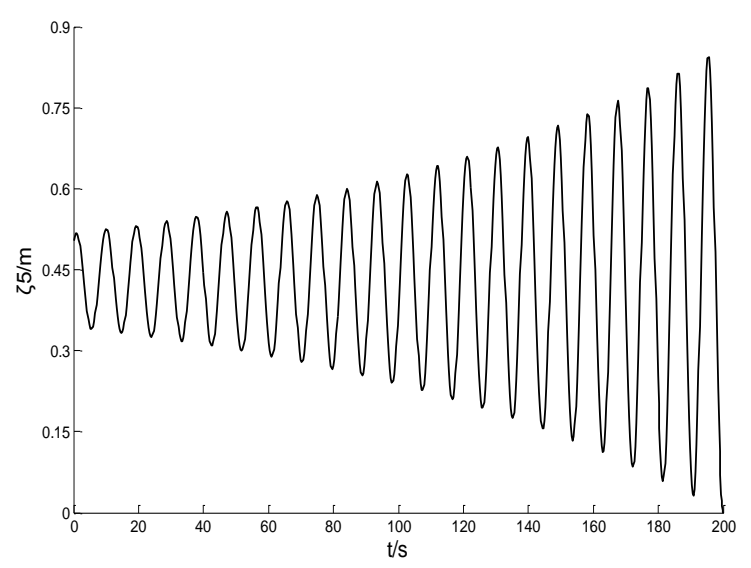

Fig. 11. A point instability time history

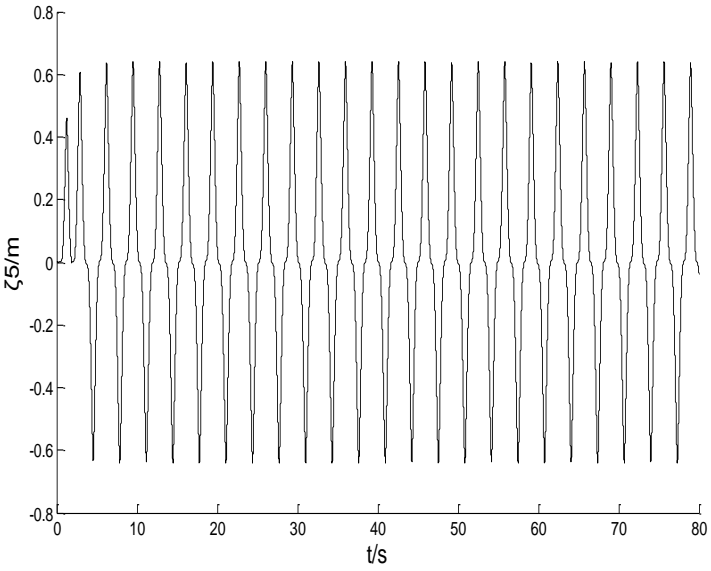

Fig. 12. B point stability time history

From Fig. 11 and Fig. 12 can be seen, the use of stability analysis method Mathieu equation, the stability of the platform mooring monitoring weak excitation parameters were analyzed, and stability in the region can be unstable region, taking regional stability and instability different spot area, time-history analysis, shows that the cycle stability of the region mooring monitoring platform in the random flow field action motion response can be a stable solution, unsteady region mooring monitoring platform in the random flow field effect movement the response can't be stable periodic solution.

\section{Conclusions}

Deformation parameters can be determined by the method of submerged monitoring platform in the frequency ratio over time and second-order curve 0,1,0.5 approximate solution, and then through the Floquet theory to study its stability characteristics under weak excitation parameters to 
determine regional stability and instability regions.

Using finite element method for flow field of structural vibration modes, combined with virtual mass ideological Act, established to consider the role of the flow field of submerged monitoring platform coupled fluid-solid finite element models to achieve a heave under the effect of the flow field, pitching modal calculations.

By changing the size of the damping system can adjust the range of the stability of the region, with the damping increases, excessive equation curve enclosed area instability range gradually reduced, gradually increasing the range of stability, so you can change the damping controlled submerge monitoring platform heave displacement, vertical roll angle range, such as external monitoring platform mooring increase helical damping side, cross-sectional shape changing platform floating body, increasing the cross-sectional diameter and the like.

\section{Acknowledgements}

This study was supported by the natural science foundation of Hubei Province project (2015CFB559),(2013CFA132)

Work partially supported by the educational commission of Hubei Province project (D20141202) and the scientific fund of China Three Gorges University project (KJ2013B042).

\section{Reference:}

[1] Kongde He, Yourong Li, Zifan Fang, Weihua Yang. Numerical Simulation of Impact Tension of Mooring Line for Submerge Monitoring Platform [J]. Vibration, Test and Diagnosis, 2013, 48 (3): 472-477.

[2] HE Kongde, LI Yourong, Fang Zifan, Yang Weihua. Calculation model and numerical simulation of dynamic response of mooring line type underwater platform [J]. China Mechanical Engineering, 2013, 23 (4):432-436.

[3] Han Cao, Yougang Tang. Design of Floating Foundation and Research on Amplitude Frequency Motion Characteristics of Semi Submerged Fan [J]. Ocean engineering, 2013, 31 (2): 61-67.

[4] Yougang Tang, Marine Engineering Structure Mechanics [M]. Tianjin: Tianjin University press, 2008.

[5] Jian Yang. The New MD Nastran Finite Element Example Tutorial [M]. Beijing: Mechanical Industry Press, 2008.

[6] Yongnian Huang. Nonlinear dynamics theory [M]. Beijing: Peking University press, 2010.

[7] Qiuhuang Dong, $\mathrm{Li}$ Chen. Dynamic Response Evaluation and Adaptive Stabilization Control and Active Suppression of Flexible Space Manipulator Based on The Capture Load [J]. Vibration and Shock, 2014, 33 (14): 101-107. 